\title{
Éclosion, au sein d'une collectivité, de rougeole contractée en voyage, Ontario 2009
}

\author{
Armstrong $\mathrm{J}^{1^{*}}$, Arajs $\mathrm{A}^{1}$ Bailey $\mathrm{N}^{1}$ et Wang $\mathrm{HL}^{1}$ \\ Service de santé publique de la région de Waterloo (Ontario) \\ Auteur-ressource : JArmstrong@regionofwaterloo.ca
}

\section{Résumé}

Contexte : Le Canada a maintenu un statut d'élimination de la rougeole depuis 1998. Cependant, des cas importés surviennent continuellement.

Objectif : Décrire les mesures de santé publique en réponse à un cas importé de rougeole dans la région de Waterloo, en Ontario, au cours du mois de mai 2009.

Résultats : Les pistes des contacts et des expositions ont été suivies et les cas ont rapidement fait l'objet d'une investigation afin d'en déterminer la source. Par des mécanismes de signalement systématiques, on a pu constater que le cas index avait probablement été exposé lors de vacances à Disney World à un cas de rougeole confirmé en laboratoire chez un garçon non vacciné âgé de 9 ans et provenant du Royaume-Uni. Le Laboratoire national de microbiologie du Canada a confirmé que le cas index était atteint de la souche D4, la même souche qui avait circulé au Royaume-Uni et dont était atteint le garçon britannique. Au total, un cas probable et six cas confirmés ont été signalés. L'âge médian des cas confirmés était de 14,5 ans (âge moyen 17 ans, âges allant de 6 à 39 ans). Cinq cas confirmés (83\%) concernaient des sujets de sexe féminin. Un cas confirmé (17\%) a été hospitalisé. Aucun décès n'a été associé à l'éclosion.

Conclusion : Cette épidémie met en évidence l'importance de la collaboration des services de soins cliniques, du laboratoire sanitaire et des services de santé publique à tous les ordres de gouvernement afin de mener une investigation et de maîtriser une éclosion de rougeole. Les voyages à l'étranger et une transmission locale continue demeureront possiblement des obstacles à l'éradication de la rougeole dans les pays développés.

\section{Introduction}

La rougeole est une infection virale aiguë dont le taux de létalité est de 1 à 3 décès pour 1000 cas. Une encéphalite aiguë survient chez environ 1 personne pour 1000 cas. La rougeole est l'une des maladies infectieuses les plus contagieuses. Elle est l'une des principales causes de décès associés aux maladies évitables par la vaccination chez les enfants, à l'échelle mondiale et particulièrement dans les pays en développement $(1,2)$. Le Canada a maintenu un statut d'élimination de la rougeole depuis 1998. À l'échelle du pays, la transmission continue a été éradiquée par les programmes de vaccination actuels, qui prévoient deux doses de vaccin contre la rougeole, et par la vaste couverture vaccinale dans la population générale. Malgré cela, des cas importés surviennent continuellement (3).

Le 25 mai 2009, un hôpital local a signalé un cas de rougeole chez une fillette non vaccinée âgée de 10 ans au Service de santé publique de la région de Waterloo. Le cas s'était présenté à l'hôpital et un pédiatre perspicace a émis l'hypothèse clinique de la rougeole. Le résultat de sérologie qui a été ensuite communiqué au Service de santé publique de la région de Waterloo, le 26 mai 2009, était positif (réactif à l'immunoglobuline M [lgM] spécifique au virus de la rougeole). Les analyses subséquentes sur des échantillons d'urine et d'écouvillonnage des yeux par la technique de transcription inverse suivie de la réaction en chaîne de la polymérase (RT-PCR) ont permis de détecter l'acide ribonucléique du virus de la rougeole et de confirmer hors de tout doute le diagnostic de rougeole. La patiente avait visité Walt Disney World en Floride, du 3 mai au 10 juin 2009. 
Cet article décrit l'investigation menée par les services de santé publique locaux et porte sur la détermination de la source de l'infection, la définition des cas, les enquêtes en laboratoire, les mesures de santé publique mise de l'avant et la communication des risques. II met en lumière l'importance de la santé publique et des mesures de lutte contre les infections, ainsi que des taux élevés de vaccination dans la collectivité et vise à prévenir efficacement la transmission et à maîtriser les éclosions.

\section{Méthodes}

\section{Identification de la source}

Le cas index a été signalé à l'Agence ontarienne de Protection et de promotion de la santé et au ministère de la Santé de l'Ontario et des Soins de longue durée, conformément aux procédures courantes.

\section{Recherche des cas et activités de collecte de données}

Les cas ont été définis selon les critères du ministère de la Santé de l'Ontario et des Soins de longue durée (Tableau 1) [4].

Tableau 1. Définitions de cas : éclosion, au sein d'une collectivité de la région de Waterloo, de rougeole contractée en voyage de mai à juin 2009

\begin{tabular}{|c|c|}
\hline Classification des cas & Définition \\
\hline Cas confirmé & $\begin{array}{l}\text { Confirmation en laboratoire de l'infection présentant des signes et symptômes concordants } \\
\text { du point de vue clinique, en l'absence d'immunisation récente par un vaccin contenant le } \\
\text { virus de la rougeole : } \\
\text { - Isolement du virus de la rougeole dans un échantillon clinique approprié (p. ex. } \\
\text { écouvillonnage, aspirat ou lavement du nasopharynx et échantillon d'urine); } \\
\text { - Détection de l'acide ribonucléique (ARN) du virus de la rougeole dans un échantillon } \\
\text { clinique approprié; } \\
\text { - Séroconversion ou augmentation marquée (par un facteur d'au moins quatre) du titre } \\
\text { des anticorps immunoglobuline G (IgG) spécifiques au virus de la rougeole dans le } \\
\text { sérum en phase de convalescence par rapport au sérum en phase aiguë, par une } \\
\text { épreuve sérologique standard; } \\
\text { - Sérologie positive pour les anticorps immunoglobuline M (IgM) spécifiques au virus de la } \\
\text { rougeole effectuée à l'aide d'une méthode recommandée chez une personne ayant un } \\
\text { lien épidémiologique avec un cas confirmé en laboratoire ou qui a voyagé récemment } \\
\text { dans une région où l'on sait qu'il y a des cas de rougeole; } \\
\text { - Signes et symptômes concordants du point de vue clinique chez une personne ayant un } \\
\text { lien épidémiologique (c.-à-d., ayant eu un contact étroit) avec un cas confirmé en } \\
\text { laboratoire. }\end{array}$ \\
\hline Cas probable & $\begin{array}{l}\text { - Signes et symptômes concordants du point de vue clinique en l'absence d'enquêtes en } \\
\text { laboratoire et en l'absence d'un lien épidémiologique avec un cas confirmé en } \\
\text { laboratoire; ou } \\
\text { - Signes et symptômes concordants du point de vue clinique chez une personne ayant } \\
\text { voyagé récemment dans une région où l'on sait qu'il y a des cas de rougeole. }\end{array}$ \\
\hline $\begin{array}{l}\text { Critères de preuve } \\
\text { clinique }\end{array}$ & $\begin{array}{l}\text { Signes et symptômes concordants du point de vue clinique caractérisés par tous les } \\
\text { éléments suivants : } \\
\text { - Fièvre supérieure ou égale à } 38,3^{\circ} \mathrm{C} \text { (voie orale) } \\
\text { - Toux, coryza ou conjonctivite (et ensuite) } \\
\text { - Éruption cutanée maculopapulaire généralisée durant au moins } 3 \text { jours. }\end{array}$ \\
\hline
\end{tabular}


Les cas ont été interrogés par les infirmières du Service de santé publique de la région de Waterloo au moyen d'un questionnaire spécifique à la rougeole leur permettant de documenter les antécédents cliniques et de vaccination des cas, ainsi que d'autres facteurs de risque.

\section{Enquête en laboratoire}

Les échantillons de tous les cas ont été prélevés par l'un ou l'autre des fournisseurs de soins de santé. Les enquêtes en laboratoire ont été effectuées au Laboratoire central de santé publique de l'Ontario à Toronto. Le génotypage a été mené au Laboratoire national de microbiologie à Winnipeg, au Manitoba.

\section{Mesures de santé publique}

Le Service de santé publique de la région de Waterloo a lancé une intense campagne de recherche de contacts du cas index, en répertoriant toutes ses activités et ses expositions au cours de la phase contagieuse.

La communication régulière avec l'équipe de lutte contre les infections nosocomiales a été maintenue, et la gestion de la liste des personnes avec qui les patients ont été en contact a été coordonnée. Le Service de santé publique de la région de Waterloo a suivi la piste des expositions dans la collectivité et dans les cabinets de médecins, et l'équipe de lutte contre les infections nosocomiales a géré les expositions à l'hôpital.

\section{Communication des risques}

Les messages de communication destinés au public et aux fournisseurs de soins de santé ont été coordonnés par le Service de santé publique de la région de Waterloo et diffusés conformément aux procédures courantes.

\section{Résultats}

\section{Identification de la source}

Le ministère de la Santé de l'Ontario et des Soins de longue durée a partagé les renseignements sur l'éclosion avec d'autres unités sanitaires en Ontario et avec l'Agence de santé publique du Canada (l'Agence). Étant donné que le cas index avait passé du temps en Floride au cours de la période d'exposition, l'Agence a contacté des unités de lutte contre les maladies aux États-Unis (Centers for Disease Control and Prevention ou CDC).

Les CDC ont ensuite informé l'Agence et le Service de santé publique de la région de Waterloo d'un cas de rougeole confirmé en laboratoire chez un garçon non vacciné âgé de 9 ans et provenant du Royaume-Uni qui avait visité Walt Disney World du $1^{\mathrm{er}}$ au 15 mai 2009. Le Service de santé publique de la région de Waterloo a comparé les itinéraires des deux cas et a relevé trois occasions de contact potentiel. Un diagramme temporel illustrant le déroulement probable de la transmission, qui montre le lien entre la souche D4 du virus de la rougeole qui circule au Royaume-Uni et le dernier cas ayant été signalé dans le cadre de l'éclosion de rougeole dans la région de Waterloo, est résumé à la Figure 1. 
Figure 1. Éclosion, au sein d'une collectivité de la région de Waterloo, de rougeole contractée en voyage de mai à juin 2009

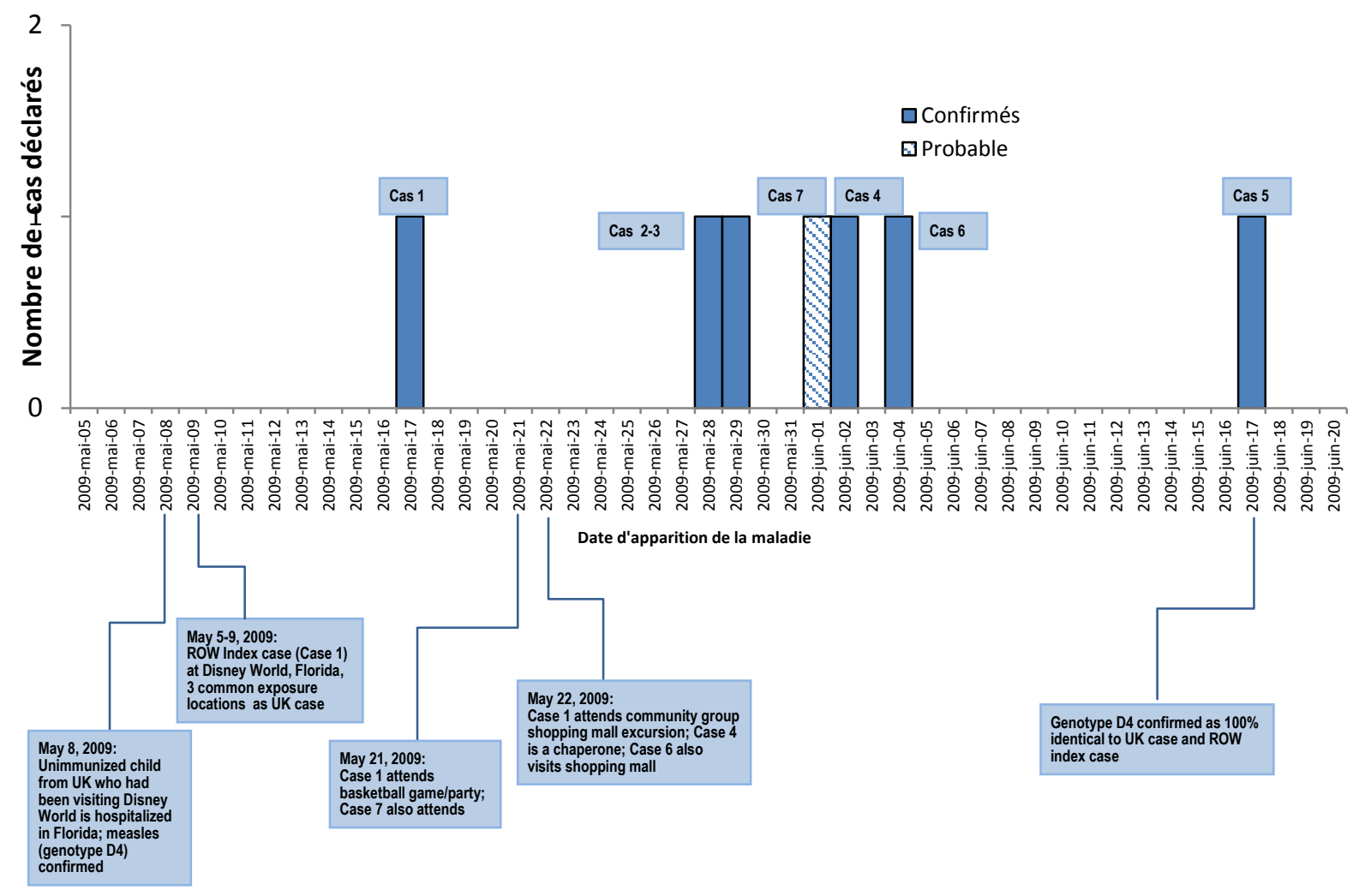

\section{Épidémiologie descriptive}

Au total, un cas probable et six cas confirmés ont été détectés (Figure 1). L'âge médian des cas confirmés était de 14,5 ans (âge moyen 17 ans, âges allant de 6 à 39 ans). Cinq cas confirmés (83 \%) concernaient des sujets de sexe féminin. Un cas confirmé (17\%) a été hospitalisé. Aucun décès n'a été associé à l'éclosion. Un résumé des cas signalés est fourni ci-dessous.

\section{Cas 1,2 et 3}

Le cas index (cas 1) concernait un sujet de sexe féminin non vacciné de 10 ans, qui s'est rendu à l'hôpital et dont la maladie a été diagnostiquée par un pédiatre astucieux. Elle est devenue très malade et a dû être hospitalisée pendant plusieurs jours, mais aucune complication ne s'est manifestée. Les symptômes comprenaient un prodrome de sept jours de toux et de coryza, suivi par l'apparition d'une fièvre intermittente, d'une éruption cutanée maculopapulaire allant du visage jusqu'au torse et d'une conjonctivite bilatérale. Le quatrième jour après le début de l'éruption cutanée, des tests visant les anticorps IgM de la rougeole ont été menés et se sont révélés positifs. Des tests RT-PCR sur des échantillons d'urine et d'écoulement oculaire effectués le dixième jour après l'apparition des symptômes se sont révélés positifs. Le génotypage de l'échantillon d'urine a été confirmé comme étant identique à celui de la souche D4 qui circulait dans le sud de l'Angleterre depuis février 2009. Une culture virale de l'éruption cutanée effectuée le dixième jour après l'apparition des symptômes s'est avérée négative. Âgés de 9 et 6 ans, deux membres d'une même fratrie non vaccinés (cas 2 et 3 ) ont présenté, respectivement, des symptômes de rougeole 14 et 15 jours après l'apparition des symptômes chez le cas index. Ces cas ont connu un parcours similaire, mais moins grave, sans conjonctivite ni besoin d'hospitalisation. Aucun autre cas de rougeole n'a été relié à cette fratrie.

\section{Cas 4}


Le cas 4 était une femme de 39 ans (née en 1970) avec un prodrome de trois jours de toux, de frissons et de fièvre, suivi d'une éruption cutanée modifiée sous forme de taches roses fines qui ne sont pas devenues confluentes et qui ont disparu en deux jours. Le 22 mai 2009, le cas 4 accompagnait en promenade dans un centre commercial un groupe communautaire dont faisait partie le cas 1. Les symptomes sont apparus le 2 juin 2009, et la rougeole a ensuite été confirmée par test sérologique. Les dossiers de vaccination indiquent que le cas 4 avait reçu le vaccin contre la rougeole à l'âge de 11 mois et le vaccin antirougeoleux, antiourlien et antirubéoleux (ROR) à l'âge de 5 ans. Le cas 4 était une infirmière qui s'occupait d'enfants médicalement fragiles dans un hôpital local. On lui a conseillé de s'isoler et de se mettre en congé. Elle n'a pas travaillé jusqu'au cinquième jour après le début de l'éruption cutanée. Les tests visant les anticorps $\lg M$ et $\lg$ de la rougeole effectuée le cinquième jour étaient positifs. L'uroculture et les tests de réaction de polymérisation en chaîne menés le huitième jour après l'apparition des symptômes se sont révélés négatifs.

\section{Cas 5 et 6}

Le 24 juin 2009, un cas suspect de rougeole chez un homme de 19 ans a été signalé au Service de santé publique de la région de Waterloo. II n'avait eu aucun contact direct connu avec le cas index. Le cas 5 était très malade et a consulté un médecin à l'hôpital. II n'a pas été admis, mais a été gardé pendant la nuit dans la salle d'urgence. Les symptômes sont apparus le 17 juin 2009 et comprenaient un prodrome de cinq jours de toux, de coryza et de fièvre, suivi d'une éruption cutanée maculopapulaire et d'une conjonctivite. Les dossiers de vaccination indiquaient qu'il avait reçu deux doses de vaccin ROR à l'âge de 9 et 10 mois dans son pays d'origine. Des tests visant les anticorps $\lg M$ et $\lg G$ de la rougeole ont été effectués le deuxième jour après l'apparition de l'éruption cutanée. Les anticorps IgM de la rougeole étaient réactifs, alors que les anticorps IgG ne l'étaient pas. Pendant sa convalescence, les tests visant les anticorps IgG de la rougeole effectués au cours du seizième jour après l'apparition des symptômes se sont révélés positifs. Les tests RT-PCR sur des écouvillons de sécrétions nasopharyngées effectués le huitième jour après l'apparition des symptômes étaient positifs, et le génotypage subséquent a confirmé une correspondance parfaite avec la souche D4 du cas index.

Pendant sa prise en charge, le cas 5 a indiqué que sa sœur jumelle (cas 6) présentait des symptômes similaires le 4 juin 2009, soit 13 jours avant l'apparition de ses symptômes à lui. Le cas 5 a également précisé que sa sœur passait beaucoup de temps au centre commercial local associé à l'éclosion. Une enquête subséquente menée par le Service de santé publique de la région de Waterloo a permis de déterminer que le cas 6 s'était effectivement rendu au centre commercial local associé à l'éclosion le 22 mai 2009. Cela a été confirmé par un reçu de transaction pour un achat effectué ce jour-là. Des tests sérologiques visant les anticorps IgM et lgG de la rougeole menée un mois après l'apparition de l'éruption cutanée ont permis de confirmer la maladie chez le cas 6 , car ils étaient tous deux positifs. Les symptômes comprenaient un prodrome de cinq jours de toux, de coryza et de fièvre, suivi d'une éruption cutanée maculopapulaire. Les dossiers de vaccination indiquaient que la sœur jumelle (cas 6) avait reçu 2 doses de vaccin ROR à l'âge de 9 et 10 mois dans son pays d'origine. Elle était très malade et a consulté un médecin à l'hôpital. Même si elle n'a pas été admise, on l'a gardée pendant la nuit dans la salle d'urgence.

Les tests RT-PCR et le génotypage du cas 5 , soit le dernier cas signalé dans le cadre de l'éclosion, ont permis de déterminer que la souche de la rougeole était la souche D4. Ce cas était donc identique au cas index de Waterloo (cas 1).

\section{Cas 7 (cas probable)}

Le cas 7 était un sujet de sexe féminin de 11 ans qui a présenté des symptômes 11 jours après l'exposition au cas index lors d'un match de basket-ball, le 21 mai 2009. Les symptômes étaient peu sévères et comprenaient une toux, un coryza et des maux de gorge, suivis par une légère fièvre et une éruption cutanée. L'éruption cutanée allait du visage jusqu'au torse, mais se présentait sous forme de petites bosses qui ont disparu en deux jours sans devenir confluentes. Le cas avait reçu une dose de vaccin ROR à l'âge de 13 mois, mais les parents ont ensuite décidé de ne pas poursuivre la vaccination. Des tests visant les anticorps $\operatorname{lgM}$ et $\lg G$ de la rougeole ont été effectués le cinquième jour après l'apparition de l'éruption cutanée. Les anticorps IgM étaient très réactifs et les anticorps IgG n'étaient pas concluants. Le test sur l'écouvillon prélevé sur la gorge le neuvième jour à partir de l'apparition des symptômes pour détecter une réaction de polymérisation en chaîne et pour la culture s'est avéré négatif. Les parents ont refusé que d'autres tests soient faits. Les chercheurs ont 
émis l'hypothèse selon laquelle il s'agissait d'un cas de rougeole modifiée, avec une excrétion virale minime due à une immunisation partielle. Même si ce cas ne correspondait techniquement pas aux lignes directrices du Ministère (4) concernant un cas probable, il a été classé en tant que tel, étant donné le fort lien épidémiologique. Un membre de la fratrie du cas 7 qui n'était pas vacciné n'a pas présenté de symptômes.

\section{Enquête en laboratoire}

Il a été confirmé que le génotypage du cas index de Waterloo (cas 1) correspondait parfaitement à celui de la souche D4 décelée chez le cas au Royaume-Uni et de la souche qui circulait dans le sud de l'Angleterre depuis février 2009.

\section{Mesures de santé publique}

Les expositions recensées pour le cas index comprenaient les salles d'attente des hôpitaux et des médecins de ville, ainsi que de nombreuses activités communautaires. Deux activités communautaires ont particulièrement attiré l'attention : un match de basket-ball, le 21 mai 2009, et une promenade de groupe communautaire dans un centre commercial local, le 22 mai 2009.

Une surveillance active a été lancée en téléphonant directement et en envoyant des courriels aux quelque 87 contacts communautaires désignés comme étant à risque. Un travailleur de la santé à risque a été trouvé dans la collectivité (cas 4). L'enquête des contacts à l'hôpital n'a décelé aucun travailleur de la santé à risque. La vaccination a fait l'objet d'une discussion avec les contacts à risque ou leurs parents et leur a été vivement recommandée. Cependant, beaucoup d'entre eux y étaient philosophiquement opposés. On a conseillé aux contacts à risque de s'isoler jusqu'à la fin de la période d'incubation potentielle, et la plupart d'entre eux se sont montrés très coopératifs. II n'y avait pas de contacts à haut risque nécessitant de l'immunoglobuline (c.-à-d. les personnes immunodéprimées, les nourrissons et les femmes enceintes).

\section{Communication des risques}

Des avis écrits périodiques ont été envoyés par télécopieur à tous les fournisseurs primaires locaux de soins de santé et les hôpitaux de la région de Waterloo pour offrir des détails sur les cas, recommander une surveillance accrue, encourager la vaccination des personnes à risque, éviter l'exposition à des contacts dans les salles d'attente et signaler les cas suspects. Un avis aux médias a été publié afin d'informer le public de la date et de la période éventuelles de l'exposition au centre commercial local visité par le cas index alors qu'il était contagieux, ainsi que d'encourager la vaccination des personnes à risque. Des lettres et des fiches d'information ont été distribuées aux familles participant au programme de basket-ball.

\section{Discussion}

Cette éclosion illustre la nature hautement contagieuse de la rougeole et le risque de transmission à l'échelle de la collectivité à partir d'un seul cas. Il y a eu une excellente collaboration entre les professionnels de santé publique locaux, provinciaux et fédéraux et une coordination optimale entre la santé publique et les établissements de soins cliniques. Les pratiques de lutte contre les infections dans les hôpitaux et les cabinets de médecins ont probablement joué un rôle dans la prévention de la transmission. Les cas 1, 5 et 6 ont tous passé du temps en milieu hospitalier pendant qu'ils étaient contagieux, mais aucune transmission ne s'est produite à l'hôpital. Les taux élevés de vaccination dans la région de Waterloo (traditionnellement de 90 à $95 \%$ chez les enfants scolarisés dans des écoles publiques de langue anglaise) ont également été un facteur clé dans la prévention de la propagation.

Les six cas confirmés lors de cette éclosion étaient non vaccinés ou mal immunisés. Les cas 4 et 7 étaient partiellement immunisés et avaient des maladies moins graves et des éruptions cutanées transitoires modifiées. Dans les deux cas, les tests de réaction de polymérisation en chaîne se sont avérés négatifs, peut-être en raison d'une excrétion virale moindre due à une immunisation partielle. Cela pourrait expliquer les anticorps $\lg G$ de rougeole très réactifs et l'absence de transmission au membre de la fratrie non vacciné du cas 7 .

Les cas 5 et 6 avaient immigré au Canada en 2005 et fréquenté l'école secondaire pendant plusieurs années en Ontario avant de déménager dans la région de Waterloo pour aller à l'université. Ils avaient chacun reçu deux vaccinations ROR dans leur pays d'origine, mais à l'âge de 9 et 10 mois. Cela démontre que les personnes immigrant au Canada en provenance de pays en développement peuvent être mal immunisées et représenter 
un groupe de priorité plus élevée. Les populations immigrées ne sont pas tenues de produire la preuve de leur vaccination avant d'entrer au Canada et peuvent être un groupe particulièrement vulnérable. Les cas 5 et 6 étaient très malades malgré une certaine immunisation, peut-être parce qu'ils avaient peu de protection, voire aucune. Les tests sérologiques pour les oreillons et la rubéole n'ont démontré aucune immunité. Comme les deux cas étaient en faveur de la vaccination, ils ont ensuite reçu deux doses du vaccin ROR en conformité avec le calendrier de vaccination de l'Ontario.

Les reçus de transaction d'achat sont souvent utilisés dans le cadre d'enquêtes sur les éclosions d'origine alimentaire afin de vérifier l'exposition à un produit alimentaire en cause. Fait intéressant, cette éclosion a démontré l'utilité d'une preuve d'achat pour relier sur le plan épidémiologique deux cas distincts de prime abord et établir un lien entre tous les cas signalés lors de cette éclosion.

\section{Conclusion}

Ce cas importé de rougeole a conduit à une éclosion de la maladie relativement faible dans la région de Waterloo, étant donné la nature hautement contagieuse de la maladie et une communauté urbaine de plus de 500000 personnes. Les facteurs ayant pu contribuer à la propagation limitée dans la collectivité comprennent un taux élevé de vaccination, une investigation de l'éclosion en temps opportun, un degré élevé d'acceptation de l'isolement (même dans les familles philosophiquement opposées à la vaccination), une communication des risques et une collaboration avec les fournisseurs de soins de santé locaux. Les déplacements internationaux pourraient continuer de représenter un défi en ce qui a trait à l'éradication de la rougeole dans les pays développés, ce qui renforce l'idée qu'il est important d'avoir des taux élevés de vaccination dans la collectivité et une vigilance constante concernant les cas sporadiques ayant le potentiel de provoquer des éclosions.

\section{Remerciements}

Les auteurs tiennent à remercier les personnes suivantes qui ont aidé à gérer et à résumer cette éclosion : le personnel infirmier en santé publique, Service de santé publique de la région de Waterloo; le $D^{r} \mathrm{~J}$. Gubbay; le $D^{r} R$. Higgins et les techniciens de laboratoire de l'Agence ontarienne de protection et de promotion de la santé (Toronto, Ontario); le Laboratoire national de microbiologie (Winnipeg, Manitoba); le $D^{r} A$. Barron, pédiatre à l'hôpital de Grand River, qui a intelligemment diagnostiqué le cas index; le $D^{r} \mathrm{~W}$. Ciccotelli et les praticiens de la prévention des infections de l'hôpital de Grand River (Kitchener, Ontario); S. Dolman et le ministère de la Santé et des Soins de longue durée de l'Ontario (Toronto, Ontario); l'Agence de la santé publique du Canada (Ottawa, Ontario); les Centers for Disease Control and Prevention (Atlanta, Géorgie); le $D^{r}$ L. Nolan, médecin conseil en santé publique, Service de santé publique de la région de Waterloo; C. Harold, superviseur de l'information et de la planification, Service de santé publique de la région de Waterloo; Jennifer Cutler, infirmière en santé publique, Service de santé publique de la région de Waterloo.

\section{Références}

(1) American Academy of Pediatrics. Red Book 2009 Report of the Committee on Infectious Diseases. $28^{\text {th }}$ ed. Elk Grove Village, IL: American Academy of Pediatrics; 2009.

(2) Mandell G., Douglas R. and Bennett J. Principles and Practices of Infectious Disease $6^{\text {th }}$ ed. Philadelphia, PA: Elsevier Churchill Livingstone; 2005.

(3) Agence de la santé publique du Canada. Guide canadien d'immunisation. $7^{\mathrm{e}}$ édition, Ottawa : Agence de la santé publique du Canada, 2006.

(4) Ministère de la Santé et des Soins de longue durée de l'Ontario. Protocole concernant les maladies infectieuses. Toronto : Imprimeur de la Reine pour l'Ontario, 2009. 